\title{
Estimation of the Flexural States of a Macro-Micro Manipulator Using Point-Acceleration Data
}

\author{
Kourosh Parsa, Jorge Angeles, and Arun K. Misra
}

\begin{abstract}
The work reported here was motivated by the development of manipulator systems comprising one long-reach, flexible-link subsystem, termed the macromanipulator, and a short-reach, rigid-link subsystem, called the micromanipulator. The flexural states of the macromanipulator, needed for controlling such systems effectively, are not usually measurable directly. For this reason, a state-estimation algorithm is proposed which uses the velocity and angular-velocity data of the micromanipulator base to estimate the flexural states of the macromanipulator. The velocity data are inferred from the acceleration signals delivered by a kinematically redundant set of triaxial accelerometers. The accelerometer signals are also utilized to calculate the translational and angular accelerations of the micromanipulator base, which are, in turn, used along with the dynamics equations of the micromanipulator to obtain the reaction force and moment acting between the two subsystems. Treating the force and the moment as inputs, the dynamics equations of the macromanipulator alone are used in the observer, thus reducing the order of the dynamics model. The state-output relations, on the other hand, are linearized in closed form to lower the computational cost. The relations thus obtained are then used in an extended Kalman filter to estimate the flexural states of the system.
\end{abstract}

Index Terms-Flexible manipulator, flexural coordinates, linearized kinematics, macro-micro manipulator, state estimation, twist vector.

\section{INTRODUCTION}

$\mathbf{K}$ INEMATICALLY redundant manipulators composed of a rigid-link, rigid-joint robot and a structurally flexible arm, on top of which the former is located, constitute a new paradigm of long-reach manipulation systems. This new paradigm motivated the ongoing research work reported here. One such manipulation system is the mobile servicing system (MSS) of the International Space Station. The MSS is composed of a mobile base; the Canadarm2 manipulator; and the special purpose dextrous manipulator (SPDM), which has recently been renamed Dextre. Canadarm2 is poised to carry, at the end of its fully extended length of $17.5 \mathrm{~m}$, a dual-arm SPDM with a total of 15 shorter links. This paper focuses on the development of state-estimators for this class of systems.

Manuscript received November 17, 2003; revised April 14, 2004 and August 5, 2004. This paper was recommended for publication by Associate Editor K. Yoshida and Editor I. Walker upon evaluation of the reviewers' comments. This work was supported by the Natural Sciences and Engineering Research Council of Canada through Research Grants OGP0004532 and OGP0000967.

K. Parsa is with the Directorate of Spacecraft Engineering, Canadian Space Agency, Saint-Hubert, QC J3Y 8 Y9 Canada (e-mail: kourosh.parsa@ space.gc.ca).

J. Angeles and A. K. Misra are with the Department of Mechanical Engineering and with the Centre for Intelligent Machines, McGill University, Montreal, QC H3A 2K6 Canada (e-mail: angeles@cim.mcgill.ca; arun.misra@ mcgill.ca).

Digital Object Identifier 10.1109/TRO.2005.844677
In this paper, flexural generalized coordinates, or flexural coordinates for short, are understood as the elements of a set of real variables that can describe the deformed shape of a structurally flexible link or system in a discretized sense. These coordinates can be, for example, the nodal displacements in a finite-element mesh, the end-point displacements of the flexible links, or the generalized coordinates used in the assumed-modes method. The generalized coordinates, along with their time rates of change, the flexural generalized velocities, constitute the flexural states of the system.

Since the very early experiments on flexible manipulators, the problem of determining the flexural states has attracted the attention of many researchers. In [1], an optical sensor-comprising a light bulb, a focusing lens, and a photodetector-was used to determine the end-point displacement of a single-link flexible manipulator, which was then used in a state-feedback control loop. The data were then processed in a discrete filter to obtain the system states. A similar instrumentation system was described in [2].

The flexible-link manipulator systems reported in [3]-[5] were instrumented by vision systems meant to measure the end-point displacement of the flexible links. However, a vision system that can capture the deformed shape of a flexiblemanipulator links in three dimensions may take a long processing time, which could make them unsuitable for real-time applications. Nevertheless, a vision system may be used every several sampling periods to correct the results of other types of sensor systems.

In [6], the authors suggested the use of three-axis force and torque sensors at the proximal end of each link to estimate the flexural states of a two-link, three-degree-of-freedom flexible manipulator using an observer. Other researchers have reported the use of strain gauges for the same purpose, among many others [7]-[10].

Another type of sensor used for the estimation of flexural states is the accelerometer. A system which kinematically resembles that of [6], and which is instrumented with three singleaxis accelerometers attached to the manipulator tip was discussed in [11], where the linear three-dimensional flexibility model of [12] was used. In both these papers, a number of generalized coordinates represented the deformation of the whole system as measured at the end effector, i.e., no discretization was involved. It is worth noting that, even if all the joints of a flexible manipulator with linearly elastic links are locked, the entire system may not behave as a linear system due to geometric nonlinearities.

In [13], the authors discussed a full-state sliding observer for a similar system. The instrumentation system included two single-axis accelerometers attached at the end of each link. The 


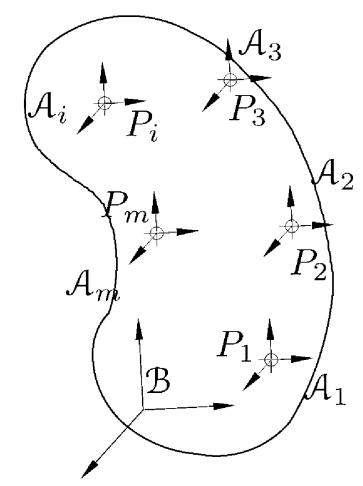

Fig. 1. Redundant set of triaxial accelerometers $\mathcal{A}_{i}$ attached to the rigid-body $\mathcal{B}$ located at points $P_{i}$, for $i=1, \ldots, m>3$.

accelerometer signals were filtered and integrated in charge amplifiers to obtain the link end-point velocity. It appears, however, that the accelerometer orientation changes induced by the system flexural motion has been neglected in all of [11]-[13]; this may result in erroneous estimation results.

The use of accelerometers, either alone or along with strain gauges, to instrument structurally flexible manipulators has been reported extensively, e.g., in [14]-[17]. However, in all these works the accelerometer signals are employed for acceleration feedback, not for estimating the flexural states.

Now, let us assume that an accelerometer array-i.e., a kinematically redundant set of more than three triaxial accelerometers - as depicted in Fig. 1, is attached to the base of the micromanipulator of a macro-micro structure, and that the array data are processed to obtain the twist-velocity and angular velocity — of the base. We report here on an algorithm which utilizes the accelerometer readouts and the twist data of the base to estimate the flexural states of the macromanipulator in an extended Kalman filter (EKF).

It should be noted that the relations between the flexural states and the twist, which are the state-output relations here, are nonlinear. However, a great number of today's well-known state-estimation techniques rely on the linearity of the above-mentioned relations. For example, the techniques discussed in [18]-[20], among many others, rely on the linearity of the state-output relations. To apply such techniques when these relations are nonlinear, one may linearize the relations and then try the particular technique using the linearized relations on the system. To this end, the calculation of the partial derivatives of the twist of the macromanipulator end-effector with respect to the states-the generalized coordinates and generalized velocities of the system-is essential.

The partial derivatives at play may, of course, be obtained by deriving the expressions for the twist, and then differentiating them symbolically. However, the drawback of such a method is that the differentiations have to be done for each manipulator separately, and that redundant algebraic manipulations and arithmetic operations are unavoidable. As an alternative, we derive, in closed form, the required relations in terms of other usually available entities, such as the robot Jacobian, its time derivative, the angular velocity, and the translational velocity. These variables are usually available in the control loop of a robot, so that the number of calculations needed will be dramatically reduced.
The structure of the observer proposed here is explained in Section II. Then, in Section III, we review some basic kinematic relations. The dynamics modeling of the system is the subject of Section IV. The discrete state-space dynamics equations are obtained in Section V. Next, the linear relations are derived in Section VI. The EKF relations are recalled in Section VII. Some simulation results are reported in Section VIII. The observability issue is discussed in Section IX, the paper concluding in Section X.

All the vectors and cross-product matrices used here are expressed in their corresponding local frames, overdots representing element-wise time derivatives. Furthermore, throughout the paper, subscripts or superscripts " $m$ " and "M"-in roman fonts-refer to the micromanipulator and the macromanipulator, respectively.

\section{StATE-Estimator Structure}

State estimation using an observer requires both a set of dynamics equations, expressing the modeled dynamics of the state variations, and a set of measurement equations, otherwise known as state-output relations. The measurement set includes algebraic relations in the states, whereas the set of dynamics equations, in continuous-time domain, comprises-usuallyordinary differential equations. Since the dynamics equations of a multilink manipulator are quite complicated, it would seem promising if the manipulator kinematic relations could be used as the modeled dynamics used in the observer, for the kinematics relations are far less involved algebraically. However, a kinematics-based observer has a major drawback: The kinematic relations, which relate the evolution of the states to the pose and twist of one or more bodies in the kinematic chain, are of a pure-integrator nature, thus exhibiting an inherently unstable behavior. Hence, such an observer is not used in this work.

The dynamics equations of the entire macro-micro system, when used as the modeled dynamics for the observer of a macro manipulator, are to be integrated online at each sampling time. This is especially expensive computationally because of the usually twice-as-large dimension of the system of differential equations for the entire macro-micro system. For this very reason, we suggest using the macromanipulator dynamics equations alone as the modeled dynamics. The idea of separating the dynamics of the macromanipulator from that of the micromanipulator has been used in the context of the vibration control of macromanipulators, e.g., in [21], [22], among others.

To be able to estimate the macro states using the micromanipulator dynamics, however, one needs to have the reaction wrench-force and moment-applied on the macromanipulator end effector by the base of the micro. This wrench can be determined if the dynamics equations of the micromanipulator, as a floating system, and the acceleration of its base are available. This approach is explained in full detail in Section IV.

\section{KINEMATIC RELATIONS}

\section{A. Twist Vector}

The twist vector of a body is understood here as a set of scalar variables that comprise the necessary and sufficient amount of information to determine the velocity field in the body. For a 


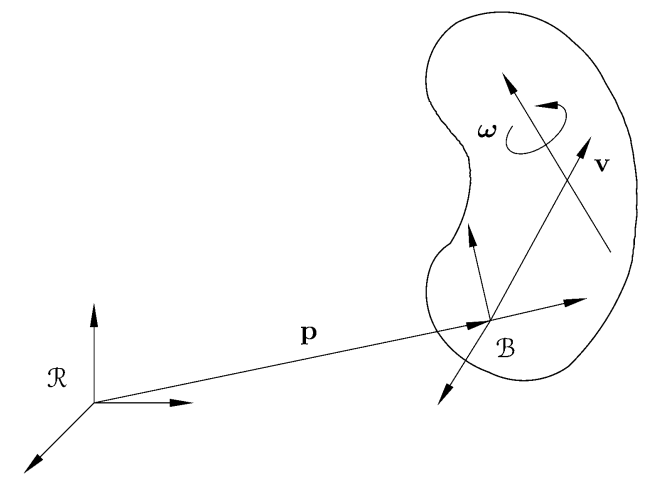

Fig. 2. Rigid-body and body and inertial coordinate frames.

rigid body, the three components of the body angular velocity and those of the velocity vector of a landmark point of the body provide this information.

We define below two different twist vectors for a rigid body: 1) vector $\mathbf{t}$, called the twist and 2) vector $\overline{\mathbf{t}}$, termed the Cartesian twist

$$
\mathbf{t} \triangleq\left[\begin{array}{c}
\dot{\mathbf{p}} \\
\omega
\end{array}\right], \quad \overline{\mathbf{t}} \triangleq\left[\begin{array}{c}
\mathbf{v} \\
\omega
\end{array}\right]
$$

where $\mathbf{p}, \mathbf{v}$, and $\boldsymbol{\omega}$, shown in Fig. 2 , are the position vector of the local-frame origin, its absolute velocity, and the frame angular velocity, respectively, all expressed in the local frame. Furthermore, according to the convention set forth in Section I, $\dot{\mathbf{p}}$ is the element-wise time derivative of vector $\mathbf{p}$; it is, in general, different from $\mathbf{v}$. In fact, we have

$$
\mathbf{v}=\dot{\mathbf{p}}+\omega \times \mathbf{p} .
$$

Hence, the twist and the Cartesian twist are related through

$$
\overline{\mathbf{t}}=\left[\begin{array}{c}
\dot{\mathbf{p}}+\omega \times \mathbf{p} \\
\omega
\end{array}\right]=\mathbf{R t}, \quad \mathbf{R} \triangleq\left[\begin{array}{cc}
1 & -\mathbf{P} \\
\mathbf{O} & 1
\end{array}\right]
$$

in which $\mathbf{P} \triangleq \operatorname{CPM}(\mathbf{p})$ is the cross-product matrix ${ }^{1}$ of vector $\mathbf{p}$, while 1 and $\mathbf{0}$ are the $3 \times 3$ identity and zero matrices, respectively.

Hence, for the micromanipulator base, for example, the twist takes on the form

$$
\mathbf{t}_{m 0} \triangleq\left[\begin{array}{ll}
\dot{\mathbf{p}}_{m 0}^{T} & \boldsymbol{\omega}_{m 0}^{T}
\end{array}\right]^{T}
$$

where $\mathbf{p}_{m 0}$ and $\boldsymbol{\omega}_{m 0}$ are the position and angular-velocity vectors of the micromanipulator base, respectively. Consequently, we have

$$
\overline{\mathbf{t}}_{m 0}=\mathbf{R}_{m 0} \mathbf{t}_{m 0}
$$

in which $\overline{\mathbf{t}}_{m 0}$ represents the Cartesian twist of the micromanipulator base, and $\mathbf{R}_{m 0}$ is the matrix $\mathbf{R}$ of (3) evaluated for the micromanipulator base with $\mathbf{P}_{m 0} \triangleq \operatorname{CPM}\left(\mathbf{p}_{m 0}\right)$, subscript zero refering to the zeroth link, i.e., the micromanipulator base.

\footnotetext{
${ }^{1}$ The cross-product matrix $\mathbf{V}$ of a vector $\mathbf{v}$, not dependent upon $\mathbf{x}$, is the skew-symmetric matrix given by,

$$
\mathbf{V} \equiv \mathrm{CPM}(\mathbf{v}) \triangleq \frac{\partial(\mathbf{v} \times \mathbf{x})}{\partial \mathbf{x}}, \quad \forall \mathbf{x} \in \mathbb{R}^{3} \Longrightarrow \mathbf{V} \mathbf{x} \equiv \mathbf{v} \times \mathbf{x} .
$$
}

\section{B. Jacobian Matrix}

In robot kinematics, the Jacobian $\mathbf{J}$ can be defined as the partial derivative of the twist vector with respect to the vector of generalized velocities, i.e.

$$
\mathbf{J} \triangleq \frac{\partial \mathbf{t}}{\partial \dot{\mathbf{q}}}
$$

where $\mathbf{q}$ represents the vector of generalized coordinates, $\dot{\mathbf{q}}$ being the vector of generalized velocities. Obviously, since the relation between $\mathbf{t}$ and $\dot{\mathbf{q}}$ is linear, the Jacobian is independent of the generalized velocities. Note that the foregoing Jacobian cannot be defined, in general, as the partial derivative of the position vector with respect to the generalized coordinates because the angular velocity is not a time derivative.

Noticing the definition of the twist vector, one can partition the Jacobian matrix into two blocks, corresponding to the two parts of the twist vector, as

$$
\mathbf{J}=\left[\begin{array}{c}
\mathbf{J}_{\dot{p}} \\
\mathbf{J}_{\omega}
\end{array}\right] ; \quad \mathbf{J}_{\dot{p}} \triangleq \frac{\partial \dot{\mathbf{p}}}{\partial \dot{\mathbf{q}}}, \quad \mathbf{J}_{\omega} \triangleq \frac{\partial \boldsymbol{\omega}}{\partial \dot{\mathbf{q}}}
$$

\section{Partial Derivatives of the Twist}

We start by recalling a basic result.

Theorem 1: The partial derivative of the angular velocity $\omega$ of a rigid-body in a serial kinematic chain with respect to the chain generalized-coordinate vector $q$ can be expressed in terms of the Jacobian $\mathbf{J}_{\omega}$, its time rate, and the cross-product matrix $\boldsymbol{\Omega}$ of the body angular velocity as

$$
\frac{\partial \boldsymbol{\omega}}{\partial \mathbf{q}}=\dot{\mathbf{J}}_{\omega}+\mathbf{\Omega} \mathbf{J}_{\omega} .
$$

The above theorem is proven in [23], where it is then used to derive closed-form relations for the partial derivatives of the Cartesian twist with respect to the generalized-coordinate and the generalized-velocity vectors. If the vector of generalized coordinates of the macromanipulator is denoted by $\psi_{M}$, based on the foregoing relations, we have

$$
\frac{\partial \overline{\mathbf{t}}_{m 0}}{\partial \dot{\boldsymbol{\psi}}_{M}}=\mathbf{R}_{m 0} \mathbf{J}_{M}, \quad \frac{\partial \overline{\mathbf{t}}_{m 0}}{\partial \boldsymbol{\psi}_{M}}=\mathbf{R}_{m 0} \dot{\mathbf{J}}_{M}+\overline{\mathbf{R}}_{m 0} \mathbf{J}_{M}
$$

in which

$$
\mathbf{J}_{M} \triangleq \frac{\partial \mathbf{t}_{m 0}}{\partial \dot{\boldsymbol{\psi}}_{M}}, \quad \overline{\mathbf{R}}_{m 0} \triangleq\left[\begin{array}{cc}
\boldsymbol{\Omega}_{m 0} & -\mathbf{P}_{m 0} \boldsymbol{\Omega}_{m 0} \\
\mathbf{O} & \boldsymbol{\Omega}_{m 0}
\end{array}\right]
$$

and $\boldsymbol{\Omega}_{m 0}$ is defined as the cross-product matrix of $\boldsymbol{\omega}_{m 0}$.

\section{DYNAMICS EQUATIONS}

The dynamics modeling of flexible manipulators has been investigated by many researchers; indeed, 105 references are cited in [24]. More references can be found in [25]. Using the results reported therein, one can derive the macromanipulator mathematical model as ${ }^{2}$

$$
\mathbf{I}_{M} \ddot{\boldsymbol{\psi}}_{M}=\boldsymbol{\tau}_{M}^{s}+\boldsymbol{\tau}_{M}^{a}-\mathbf{J}_{M}^{T} \mathbf{w}_{m 0}^{e}
$$

${ }^{2}$ No matter how accurately the macromanipulator dynamics is modeled, the model can be cast in the form of (11). Therefore, there is no limitation to be attributed to the model used herein. 
in which $\mathbf{I}_{M}$ is the macromanipulator mass matrix; $\boldsymbol{\tau}_{M}^{s}$ represents the system wrench, containing all the Coriolis, gravity, centrifugal, and flexural generalized forces; $\boldsymbol{\tau}_{M}^{a}$ is the vector of actuation generalized forces; and $\mathbf{w}_{m 0}^{e}$ is the reaction wrench applied on the micromanipulator base by the end-link of the macromanipulator. Furthermore, if $\boldsymbol{\psi}_{M}$ is defined as

$$
\boldsymbol{\psi}_{M} \triangleq\left[\begin{array}{ll}
\boldsymbol{\theta}_{M}^{T} & \boldsymbol{\zeta}^{T}
\end{array}\right]^{T}
$$

where $\boldsymbol{\theta}_{M}$ and $\boldsymbol{\zeta}$ are the vectors of joint variables and flexural generalized coordinates of the macromanipulator, respectively, then

$$
\boldsymbol{\tau}_{M}^{a}=\left[\begin{array}{ll}
\boldsymbol{\tau}_{M}^{T} & \mathbf{0}_{f}^{T}
\end{array}\right]^{T}
$$

with $\boldsymbol{\tau}_{M}$ and $\mathbf{0}_{f}$ being the vector of joint-actuation torques and the zero vector of dimension $f$, the number of flexural generalized coordinates, respectively.

As mentioned in Section II, the reaction wrench $\mathbf{w}_{m 0}^{e}$ is needed if the model (11) is to be used as the modeled dynamics in the estimator. To calculate this wrench, the acceleration measurements delivered by the accelerometer array, which is installed on the micromanipulator base, are collected at each instant. In general, accelerometers are also affected by gravity; as explained in [26], however, this effect is eliminated from the acceleration differences if the accelerometers relative orientations are known. Furthermore, the accelerometer data can be processed to obtain the twist and the angular acceleration of the base, as well as the translational acceleration of the origin of its local frame. The calculation of the translational acceleration, however, requires the attitude of the micromanipulator base to eliminate the effect of gravity; that attitude can be estimated from the macromanipulator generalized coordinates and its kinematics.

The information thus obtained is substituted into the dynamics model of the micromanipulator to obtain the reaction wrench sought. This approach is formulated below. First, we notice that the dynamics model of the micromanipulator, as a rigid robot with moving base, can be derived as [27]

$$
\mathbf{I}_{m} \dot{\mathbf{v}}_{m}=\boldsymbol{\tau}_{m}^{s}+\tau_{m}^{a}+\tau_{m 0}^{e}+\boldsymbol{\tau}_{m}^{e e}
$$

in which $\mathbf{I}_{m}$ is the mass matrix, while $\boldsymbol{\tau}_{m}^{s}, \boldsymbol{\tau}_{m}^{a}$, and $\boldsymbol{\tau}_{m}^{e e}$ represent the system, actuation, and end-effector external wrenches of the micromanipulator, respectively. The system wrench includes all the Coriolis, centrifugal, and gravity forces. Moreover, the vectors $\mathbf{v}_{m}, \boldsymbol{\tau}_{m}^{a}$, and $\boldsymbol{\tau}_{m 0}^{e}$ are given by

$$
\mathbf{v}_{m}=\left[\begin{array}{c}
\mathbf{t}_{m 0} \\
\dot{\boldsymbol{\theta}}_{m}
\end{array}\right], \quad \boldsymbol{\tau}_{m}^{a}=\left[\begin{array}{c}
\mathbf{0}_{6} \\
\boldsymbol{\tau}_{m}
\end{array}\right], \quad \boldsymbol{\tau}_{m 0}^{e}=\left[\begin{array}{c}
\mathbf{w}_{m 0}^{e} \\
\mathbf{0}_{n_{m}}
\end{array}\right]
$$

where $\boldsymbol{\theta}_{m}, \boldsymbol{\tau}_{m}$, and $\mathbf{0}_{n_{m}}$ are the vectors of the micromanipulator joint variables and joint-actuation torques, and the $n_{m}$-dimensional zero vector, respectively, $n_{m}$ being the number of the micromanipulator joints.

Then, partitioning the mass matrix and all the vectors of the dynamics model (14) corresponding to the two parts of $\mathbf{v}_{m}$, we can rewrite the dynamics model as

$$
\begin{aligned}
\mathbf{M}_{b b}^{m} \dot{\mathbf{t}}_{m 0}+\mathbf{M}_{b r}^{m} \ddot{\boldsymbol{\theta}}_{m} & =\boldsymbol{\tau}_{m b}^{s}+\boldsymbol{\tau}_{m b}^{e e}+\mathbf{w}_{m 0}^{e} \\
\left(\mathbf{M}_{b r}^{m}\right)^{T} \dot{\mathbf{t}}_{m 0}+\mathbf{M}_{r r}^{m} \ddot{\boldsymbol{\theta}}_{m} & =\boldsymbol{\tau}_{m r}^{s}+\boldsymbol{\tau}_{m r}^{e e}+\boldsymbol{\tau}_{m}
\end{aligned}
$$

in which $\mathbf{M}_{b b}^{m}, \mathbf{M}_{b r}^{m}$, and $\mathbf{M}_{r r}^{m}$ are the blocks of the mass matrix $\mathbf{I}_{m} ; \boldsymbol{\tau}_{m b}^{s}$ and $\boldsymbol{\tau}_{b r}^{s}$ are the subarrays of its system wrench; and $\boldsymbol{\tau}_{m b}^{e e}$ and $\tau_{b r}^{e e}$ are the subarrays of the end-effector external wrench, i.e.

$$
\begin{aligned}
\mathbf{I}_{m} & \equiv\left[\begin{array}{cc}
\mathbf{M}_{b b}^{m} & \mathbf{M}_{b r}^{m} \\
\left(\mathbf{M}_{b r}^{m}\right)^{T} & \mathbf{M}_{r r}^{m}
\end{array}\right], \quad \boldsymbol{\tau}_{m}^{s} \equiv\left[\begin{array}{c}
\boldsymbol{\tau}_{m b}^{s} \\
\boldsymbol{\tau}_{m r}^{s}
\end{array}\right] \\
\boldsymbol{\tau}_{m}^{e e} & \equiv\left[\begin{array}{c}
\boldsymbol{\tau}_{m b}^{e e} \\
\boldsymbol{\tau}_{m r}^{e e}
\end{array}\right] .
\end{aligned}
$$

Subscripts " $b$ " and " $r$ " above refer to the base and the joint (rotational) degrees of freedom of the micromanipulator, respectively.

Next, one can solve (16b) and (16a) for $\mathbf{M}_{r r}^{m} \ddot{\boldsymbol{\theta}}_{m}$ and $\mathbf{w}_{m 0}^{e}$, respectively, to obtain

$$
\begin{aligned}
\mathbf{M}_{r r}^{m} \ddot{\boldsymbol{\theta}}_{m} & =-\left(\mathbf{M}_{b r}^{m}\right)^{T} \dot{\mathbf{t}}_{m 0}+\boldsymbol{\tau}_{m r}^{s}+\boldsymbol{\tau}_{m r}^{e}+\boldsymbol{\tau}_{m} \\
\mathbf{w}_{m 0}^{e} & =\mathbf{M}_{b b}^{m} \dot{\mathbf{t}}_{m 0}+\mathbf{M}_{b r}^{m} \ddot{\boldsymbol{\theta}}_{m}-\boldsymbol{\tau}_{m b}^{s}-\boldsymbol{\tau}_{m b}^{e e}
\end{aligned}
$$

It is worth mentioning that symbolic expressions for the reaction force and moment have been reported elsewhere, in [28], for example. However, the expressions in [28] are given in terms of the generalized coordinates, velocities, and accelerations of the entire system. Moreover, it is assumed therein that all macromanipulator joints are locked, which in not needed in our approach.

Finally, if the translational and angular accelerations of the micromanipulator base as well as its twist are determined using the accelerometer array, one can readily calculate $\dot{\mathrm{t}}_{m 0}$. As for the other information needed for the right-hand sides of (17), the subarrays of the micromanipulator system wrench $\boldsymbol{\tau}_{m b}^{s}$ and $\boldsymbol{\tau}_{m r}^{s}$ can be calculated from the dynamics equations using the joint angles and joint rates; the external wrench applied on the micro end effector is inferred from the end-effector force and torque, either known or measured directly; and the actuation torques are also assumed to be either known from the control system or measured. Thereafter, solving the above linear equations sequentially to obtain the reaction wrench is straightforward.

\section{State-Space Dynamics Model}

Let us denote the state vector of the macromanipulator by $\mathbf{x}$, which is given by

$$
\mathbf{x} \triangleq\left[\begin{array}{c}
\mathbf{x}^{1} \\
\mathbf{x}^{2}
\end{array}\right], \quad \mathbf{x}^{1} \triangleq \boldsymbol{\psi}_{M} \equiv\left[\begin{array}{c}
\boldsymbol{\theta}_{M} \\
\boldsymbol{\zeta}
\end{array}\right], \quad \mathbf{x}^{2} \triangleq \dot{\boldsymbol{\psi}}_{M} \equiv\left[\begin{array}{c}
\dot{\boldsymbol{\theta}}_{M} \\
\dot{\boldsymbol{\zeta}}
\end{array}\right]
$$

Using the above definitions of the states, one can write the mathematical model (11) of the macromanipulator in state-space form as

$$
\begin{aligned}
& \dot{\mathbf{x}}^{1}=\mathbf{x}^{2} \\
& \dot{\mathbf{x}}^{2}=\mathbf{f}(\mathbf{x})+\mathbf{B}(\mathbf{x}) \mathbf{u}(t)
\end{aligned}
$$

with $\mathbf{f}(\mathbf{x}), \mathbf{B}(\mathbf{x})$, and $\mathbf{u}(t)$ defined as

$$
\begin{aligned}
\mathbf{f}(\mathbf{x}) & \triangleq \mathbf{H}\left(\mathbf{x}^{1}\right) \boldsymbol{\tau}_{M}^{s}(\mathbf{x}) \\
\mathbf{B}(\mathbf{x}) & \triangleq\left[\begin{array}{ll}
\mathbf{H}_{1} & -\mathbf{H} \mathbf{J}_{M}^{T}
\end{array}\right] \\
\mathbf{u}(t) & \triangleq\left[\begin{array}{c}
\boldsymbol{\tau}_{M} \\
\mathbf{w}_{m 0}^{e}
\end{array}\right]
\end{aligned}
$$


in which $\mathbf{J}_{M}$ was defined in (10), while matrix $\mathbf{H}$, defined as the inverse of the mass matrix $\mathbf{I}_{M}$, is partitioned into two blocks

$$
\mathbf{H} \equiv\left[\begin{array}{ll}
\mathbf{H}_{1} & \mathbf{H}_{2}
\end{array}\right] \triangleq \mathbf{I}_{M}^{-1} .
$$

The first block $\mathbf{H}_{1}$ is an $N_{M} \times n_{M}$ matrix, with $N_{M}$ and $n_{M}$ denoting the degree of freedom of the macromanipulator and its number of links, respectively.

Hence, the discrete-time linearized form of (19) can be written as

$$
\begin{aligned}
& \mathbf{x}_{k+1}^{1}=\mathbf{x}_{k}^{1}+h \mathbf{x}_{k}^{2}+\mathbf{m}_{k}^{1} \\
& \mathbf{x}_{k+1}^{2}=\mathbf{x}_{k}^{2}+\left.h \frac{\partial \mathbf{f}}{\partial \mathbf{x}}\right|_{\hat{\mathbf{x}}_{k}} \mathbf{x}_{k}+\overline{\mathbf{u}}_{k}+\mathbf{m}_{k}^{2}
\end{aligned}
$$

in which $h$ is the sampling period, $\mathbf{m}_{k}^{1}$ and $\mathbf{m}_{k}^{2}$ are the subarrays of the vector $\mathbf{m}_{k}$ of the uncorrelated white-noise processes representing the unmodeled dynamics, and $\overline{\mathbf{u}}_{k}$ is a redefined input function given by

$$
\overline{\mathbf{u}}_{k} \triangleq h\left[\mathbf{f}\left(\hat{\mathbf{x}}_{k}\right)-\left.\frac{\partial \mathbf{f}}{\partial \mathbf{x}}\right|_{\hat{\mathbf{x}}_{k}} \hat{\mathbf{x}}_{k}+\mathbf{B}\left(\hat{\mathbf{x}}_{k}\right) \mathbf{u}_{k}\right] .
$$

Thus, in standard form, we have

$$
\mathbf{x}_{k+1}=\mathbf{A}_{k} \mathbf{x}_{k}+\tilde{\mathbf{u}}_{k}+\mathbf{m}_{k}
$$

with $\mathbf{A}_{k}$ and $\tilde{\mathbf{u}}_{k}$ defined as

$$
\begin{aligned}
& \mathbf{A}_{k} \triangleq\left[\begin{array}{cc}
1 & h \mathbf{1} \\
h\left(\frac{\partial \mathbf{f}}{\partial \mathbf{x}^{1}}\right) & \mathbf{1}+h\left(\frac{\partial \mathbf{f}}{\partial \mathbf{x}^{2}}\right)
\end{array}\right]_{\mathbf{x}=\hat{\mathbf{x}}_{k}} \\
& \tilde{\mathbf{u}}_{k} \triangleq\left[\begin{array}{ll}
\mathbf{0}_{N_{M}}^{T} & \overline{\mathbf{u}}_{k}^{T}
\end{array}\right]^{T} .
\end{aligned}
$$

To complete the derivation of the state-space dynamics model, the partial derivatives of $\mathbf{f}$ with respect to $\mathbf{x}^{1}$ and $\mathbf{x}^{2}$ must be found. Between the two, however, it is the partial derivative of $\mathbf{f}$ with respect to $\mathbf{x}^{1}$ that needs extra attention, because of the dependence of $\mathbf{H}$ on the generalized coordinates. To obtain this partial derivative, we notice from (20) that

$$
\frac{\partial \mathbf{f}}{\partial \mathbf{x}^{1}}=\left.\frac{\partial(\mathbf{H a})}{\partial \mathbf{x}^{1}}\right|_{\mathbf{a}=\boldsymbol{\tau}_{M}^{s}}+\mathbf{H} \frac{\partial \boldsymbol{\tau}_{M}^{s}}{\partial \mathbf{x}^{1}}
$$

where $\mathbf{a}$ is a dummy variable. However, the first term on the right-hand side can be written as

$$
\begin{aligned}
\left.\frac{\partial(\mathbf{H a})}{\partial \mathbf{x}^{1}}\right|_{\mathbf{a}=\boldsymbol{\tau}_{M}^{s}} & =\left.\frac{\partial\left(\mathbf{H I}_{M} \mathbf{H a}\right)}{\partial \mathbf{x}^{1}}\right|_{\mathbf{a}=\boldsymbol{\tau}_{M}^{s}} \\
& =\left.2 \frac{\partial(\mathbf{H a})}{\partial \mathbf{x}^{1}}\right|_{\mathbf{a}=\boldsymbol{\tau}_{M}^{s}}+\left.\mathbf{H} \frac{\partial\left(\mathbf{I}_{M} \mathbf{a}\right)}{\partial \mathbf{x}^{1}}\right|_{\mathbf{a}=\mathbf{f}}
\end{aligned}
$$

from which we can readily conclude that

$$
\left.\frac{\partial(\mathbf{H a})}{\partial \mathbf{x}^{1}}\right|_{\mathbf{a}=\boldsymbol{\tau}_{M}^{s}}=-\left.\mathbf{H} \frac{\partial\left(\mathbf{I}_{M} \mathbf{a}\right)}{\partial \mathbf{x}^{1}}\right|_{\mathbf{a}=\mathbf{f}} .
$$

Hence, using (29) and (31), one can derive the partial derivative of $\mathbf{f}$ with respect to $\mathbf{x}^{1}$ as

$$
\frac{\partial \mathbf{f}}{\partial \mathbf{x}^{1}}=\mathbf{H}\left(\frac{\partial \boldsymbol{\tau}_{M}^{s}}{\partial \boldsymbol{\psi}_{M}}-\left.\frac{\partial\left(\mathbf{I}_{M} \mathbf{a}\right)}{\partial \boldsymbol{\psi}_{M}}\right|_{\mathbf{a}=\mathbf{f}}\right)
$$

while the partial derivative of $\mathbf{f}$ with respect to $\mathbf{x}^{2}$ is clearly given by

$$
\frac{\partial \mathbf{f}}{\partial \mathbf{x}^{2}}=\mathbf{H} \frac{\partial \boldsymbol{\tau}_{M}^{s}}{\partial \dot{\boldsymbol{\psi}}_{M}}
$$

\section{State-Output Relations}

To obtain the state-output relations for the system, we denote the output vector by $\mathbf{y}$ and define it as

$$
\mathbf{y} \triangleq\left[\begin{array}{l}
\mathbf{y}^{1} \\
\mathbf{y}^{2}
\end{array}\right], \quad \mathbf{y}^{1} \triangleq\left[\begin{array}{c}
\boldsymbol{\theta}_{M} \\
\dot{\boldsymbol{\theta}}_{M}
\end{array}\right], \quad \mathbf{y}^{2} \triangleq \overline{\mathbf{t}}_{m 0}
$$

where $\overline{\mathbf{t}}_{m 0}$, as mentioned before, is the Cartesian twist of the micromanipulator base. While the relation of $\mathbf{y}^{1}$ with the states of the system is evidently linear and readily known, for the entries of $\mathbf{y}^{1}$ are among the system states themselves, the state-output relations pertaining to $\mathbf{y}^{2}$ are indeed nonlinear and should, thus, be linearized. To linearize these relations, $\mathbf{y}^{2}$ is expanded using a Taylor series up to first-order terms about $\hat{\mathbf{x}}$. Neglecting the higher order terms, we obtain

$$
\mathbf{y}^{2}(\mathbf{x}) \approx \mathbf{y}^{2}(\hat{\mathbf{x}})+\left.\frac{\partial \mathbf{y}^{2}}{\partial \mathbf{x}}\right|_{\hat{\mathbf{x}}}(\mathbf{x}-\hat{\mathbf{x}})
$$

where $\hat{\mathbf{X}}$ represents the estimated state vector, and, obviously

$$
\frac{\partial \mathbf{y}^{2}}{\partial \mathbf{x}} \equiv\left[\begin{array}{ll}
\frac{\partial \mathbf{y}^{2}}{\partial \mathbf{x}^{1}} & \frac{\partial \mathbf{y}^{2}}{\partial \mathbf{x}^{2}}
\end{array}\right]=\left[\begin{array}{ll}
\frac{\partial \overline{\mathbf{t}}_{m 0}}{\partial \psi_{M}} & \frac{\partial \overline{\mathbf{t}}_{m 0}}{\partial \dot{\psi}_{M}}
\end{array}\right] .
$$

The partial derivatives needed in (35) are available in closed form from (9).

Consequently, upon defining a new output vector, denoted by $\overline{\mathbf{y}}$, as

$$
\overline{\mathbf{y}} \triangleq \mathbf{y}(\mathbf{x})-\mathbf{y}(\hat{\mathbf{x}})+\left.\frac{\partial \mathbf{y}}{\partial \mathbf{x}}\right|_{\hat{\mathbf{x}}} \hat{\mathbf{x}}
$$

the state-output relation can be rewritten in the linearized form as

$$
\begin{aligned}
\overline{\mathbf{y}} & =\mathbf{C x}+\mathbf{n}(t) \\
\mathbf{C} & \triangleq\left[\begin{array}{cc}
\mathbf{E}_{n_{M} \times N_{M}} & \mathbf{O}_{n_{M} \times N_{M}} \\
\mathbf{R}_{m 0} \dot{\mathbf{J}}_{M}+\overline{\mathbf{R}}_{m 0} \mathbf{J}_{M} & \mathbf{R}_{m 0} \mathbf{J}_{M}
\end{array}\right]
\end{aligned}
$$

in which $\mathbf{n}(t)$ is the vector of the uncorrelated, white measurement-noise processes, ${ }^{3}$ and $\mathbf{E}_{n_{M} \times N_{M}}$ is a rectangular array of ones and zeros in which the largest left-hand-side block is the $n_{M} \times n_{M}$ identity matrix.

\footnotetext{
${ }^{3} \mathrm{~A}$ more realistic model for noise on the translational-velocity part of the output, which is inferred from the accelerometer-array signals by integration, will perhaps be a Markov process [29] if the accelerometer noise is assumed to be white.
} 


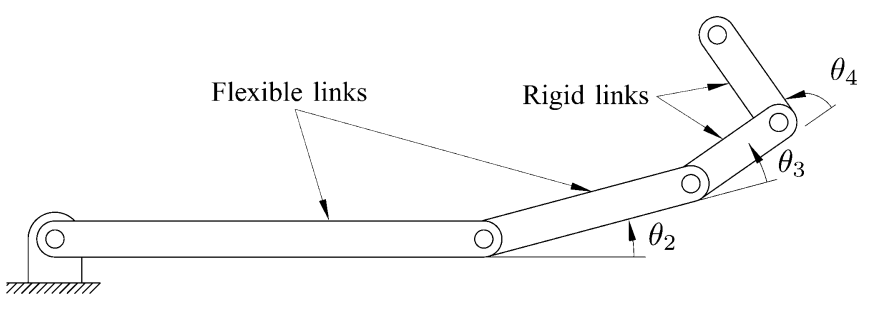

Fig. 3. Planar $R R R R$ macro-micro manipulator in its initial posture.

Hence, the time-discretized form of the state-output relation (37) can readily be written as

$$
\overline{\mathbf{y}}_{k}=\mathbf{C}_{k} \mathbf{x}_{k}+\mathbf{n}_{k}
$$

As mentioned before, using the accelerometer-array signals, one can readily calculate the twist rate- the acceleration and the angular acceleration — of the micromanipulator base. These data may also be taken as outputs. However, taking the twist-rate elements as outputs will require differentiating the dynamics equations of the flexible manipulator, which, in turn, complicates the linearization of the state-space form of the mathematical model further. Therefore, this is avoided. Nevertheless, if, in a particular problem, taking only the twist does not produce reliable results, one can include the twist-rate in the outputs and use the linearized state-output relations derived in [23].

\section{EXTENDED KALMAN FILTER}

With the linearized governing equations available, one can use the EKF relations to obtain the state estimates. These relations are derived in [30] as

$$
\begin{aligned}
\mathbf{P}_{0,0} & =\operatorname{Var}\left(\mathbf{x}_{0}\right), \quad \hat{\mathbf{x}}_{0}=E\left(\mathbf{x}_{0}\right) \\
\mathbf{P}_{k, k-1} & =\mathbf{A}_{k-1} \mathbf{P}_{k-1} \mathbf{A}_{k-1}^{T}+\boldsymbol{\Gamma}_{k-1} \mathbf{Q}_{k-1} \boldsymbol{\Gamma}_{k-1}^{T} \\
\hat{\mathbf{x}}_{k \mid k-1}^{1} & =\hat{\mathbf{x}}_{k-1}^{1}+h \hat{\mathbf{x}}_{k-1}^{2} \\
\hat{\mathbf{x}}_{k \mid k-1}^{2} & =\hat{\mathbf{x}}_{k-1}^{2}+h\left[\mathbf{f}\left(\hat{\mathbf{x}}_{k-1}\right)+\mathbf{B}\left(\hat{\mathbf{x}}_{k-1}\right) \mathbf{u}_{k-1}\right] \\
\mathbf{G}_{k} & =\mathbf{P}_{k, k-1} \mathbf{C}_{k}^{T}\left(\mathbf{C}_{k} \mathbf{P}_{k, k-1} \mathbf{C}_{k}^{T}+\mathbf{R}_{k}\right)^{-1} \\
\mathbf{P}_{k} & =\left(\mathbf{1}-\mathbf{G}_{k} \mathbf{C}_{k}\right) \mathbf{P}_{k, k-1} \\
\hat{\mathbf{x}}_{k} & =\hat{\mathbf{x}}_{k \mid k-1}+\mathbf{G}_{k}\left(\mathbf{y}_{k}-\hat{\mathbf{y}}_{k \mid k-1}\right)
\end{aligned}
$$

where $\mathbf{Q}_{k-1}$ and $\mathbf{R}_{k}$ are the covariance matrices of the uncorrelated white-noise processes $\mathbf{m}_{k-1}$ and $\mathbf{n}_{k}$, respectively.

\section{SimULATION STUDY}

The ideas explained here regarding the estimation of the flexural states using an accelerometer array are demonstrated by simulating the dynamics of a planar $R R R R$ manipulator, illustrated in Fig. 3, on a horizontal plane. The first two links of this manipulator-constituting the macromanipulator-are assumed flexible, while the other two-making up the micromanipulator-are assumed rigid. Moreover, we discretize the flexible links using the assumed-modes method, taking the clamped-free eigenfunctions as the shape-functions; the bending of each of the macromanipulator links is described by one flexural generalized coordinate. The specifications of the links are given in Table I.
TABLE I

LINK SPECIFICATIONS OF THE $R R R R$ MANIPULATOR

\begin{tabular}{l|c|c|c|c}
\hline & $\#$ & Mass (kg) & Length $(\mathrm{m})$ & $E I\left(\mathrm{Nm}^{2}\right)$ \\
\hline \multirow{2}{*}{ Macro } & 1 & 1.0 & 1.0 & 93.266 \\
\cline { 2 - 5 } & 2 & 0.50 & 0.50 & 93.266 \\
\hline \multirow{2}{*}{ Micro } & 1 & 0.20 & 0.25 & - \\
\cline { 2 - 5 } & 2 & 0.20 & 0.25 & - \\
\hline
\end{tabular}

Furthermore, it is assumed that the noise covariances are constant and given by

$$
\begin{aligned}
& \mathbf{R}=\operatorname{diag}\left(4.0 \times 10^{-3} \mathbf{1}_{4}, 1.3 \mathbf{1}_{4}, 2.5 \mathbf{1}_{2}, 0.10\right) \times 10^{-3} \\
& \mathbf{Q}=\operatorname{diag}\left(0.1 \mathbf{1}_{4}, 10^{-2} \mathbf{1}_{2}, \mathbf{1}_{4}, 10^{-2} \mathbf{1}_{2}\right) \times 10^{-5}
\end{aligned}
$$

where $\mathbf{1}_{2}$ and $\mathbf{1}_{4}$ are the $2 \times 2$ and $4 \times 4$ identity matrices, respectively. The entries of $\mathbf{R}$ are determined based on a noise-tosignal ratio of approximately $2.5 \%$ for the first three blocks and double that for the last two blocks - to account for the linearization error-whereas the entries of $\mathbf{Q}$ are guessed values. Our simulations have shown that the state-estimation results remain acceptable over a rather large range of values.

Two different manipulator motions are considered here; in each of the two cases, flexural motion is induced in the macromanipulator by having one or more of the joints to follow periodic motions. In the first case, to keep the motion simple, we assume that joints 1,2 , and 4 are locked at $\theta_{1}=0, \theta_{2}=\pi / 12 \mathrm{rad}$, and $\theta_{4}=\pi / 2 \mathrm{rad}$, and the motion of the third joint is given by

$$
\theta_{3}=\left[\frac{\pi}{8} \cos (\pi t)+\frac{\pi}{12} \cos (2)\right] u(t)
$$

where $u(t)$ is the unit step function.

Applying our algorithm, we can estimate both the flexural states and the joint-rates, but we mainly focus here on the former. Fig. 4 shows the actual and the estimated flexural generalized coordinates; the results pertaining to the flexural generalized velocities are displayed in Fig. 5. The estimation errors of the two are plotted in Figs. 6 and 7. The states with a subscript 1 pertain to the first link, while those having a subscript 2 pertain to the second link.

For the second manipulator motion, the joints 1, 2, and 4 are assumed to follow:

$$
\begin{aligned}
& \theta_{1}=\frac{\pi}{18} \sin \left(\frac{\pi}{18} t\right) u(t) \\
& \theta_{2}=\frac{\pi}{18}\left[3+\sin \left(\frac{\pi}{18} t\right)\right] u(t) \\
& \theta_{4}=\frac{\pi}{2} \cos (\pi t) u(t)
\end{aligned}
$$

while the motion of the third joint remains the same. The estimation results of the flexural coordinates and flexural generalized velocities are shown in Figs. 8 and 9, respectively, and the estimation errors are plotted in Figs. 10 and 11.

As seen from the graphs, the estimator has been able to estimate the flexural states with a high accuracy, as the theoretical and the estimated values of the flexural states are almost indistinguishable. However, a caveat is in order here. For the simulations, the "actual flexural dynamics" of the macromanipulator 


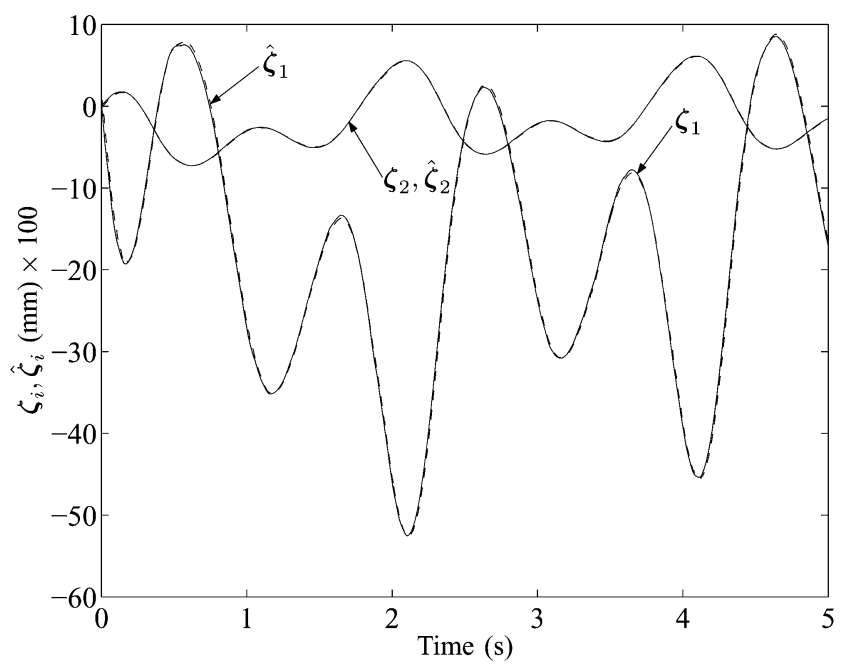

Fig. 4. Flexural generalized coordinates; (solid) theoretical values and (dashed) estimated values.

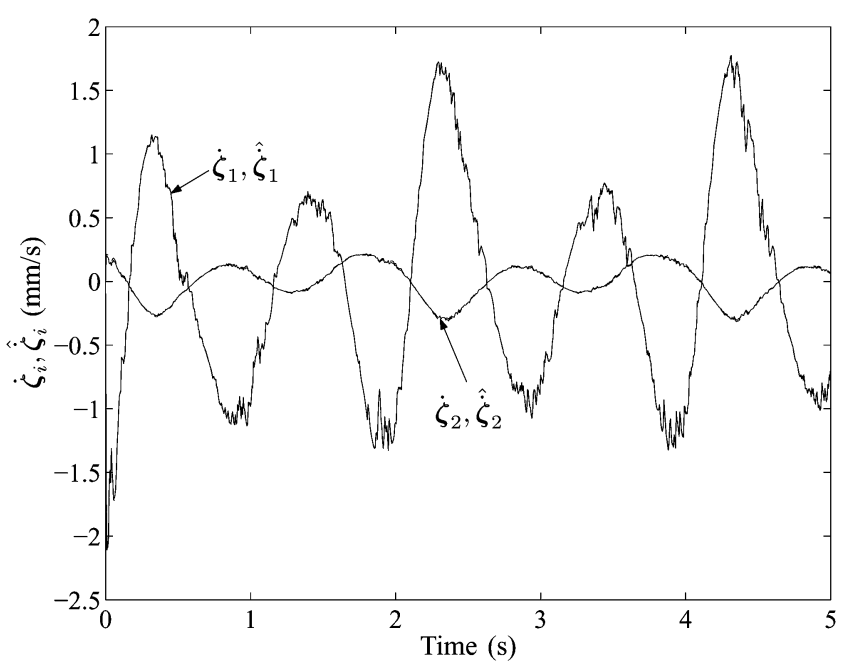

Fig. 5. Flexural generalized velocities; (solid) theoretical values and (dashed) estimated values.

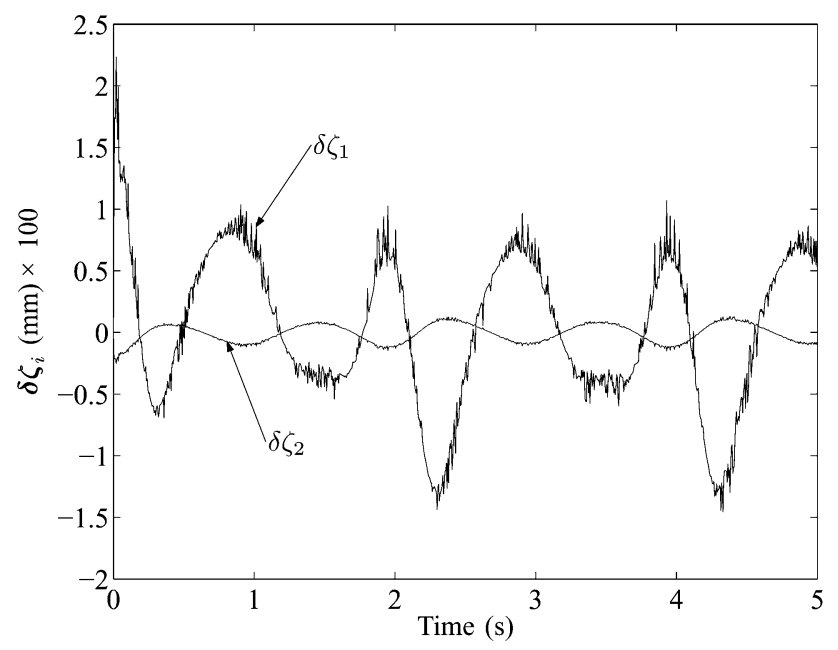

Fig. 6. Estimation error of the flexural coordinates.

has been modeled by taking one flexural coordinate for each of the flexible links, which is the same number of flexural coordinates used in the modeled dynamics employed in the state

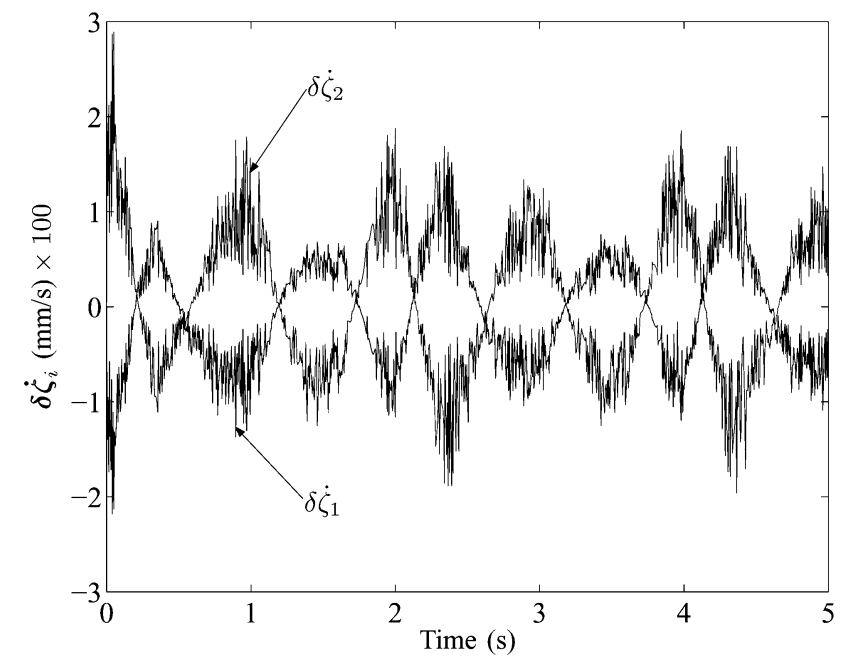

Fig. 7. Estimated error of the flexural generalized velocities.

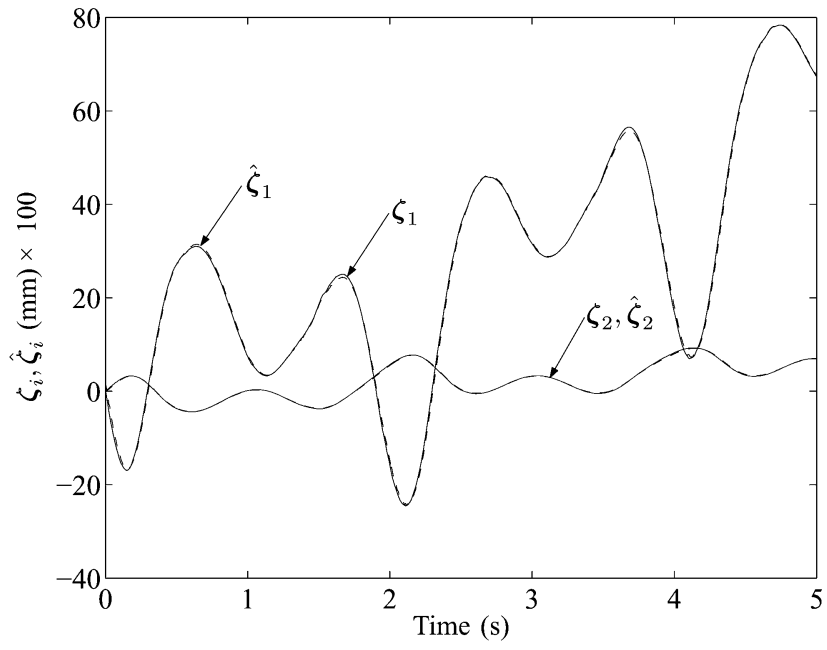

Fig. 8. Flexural generalized coordinates; (solid) theoretical values and (dashed) estimated values.

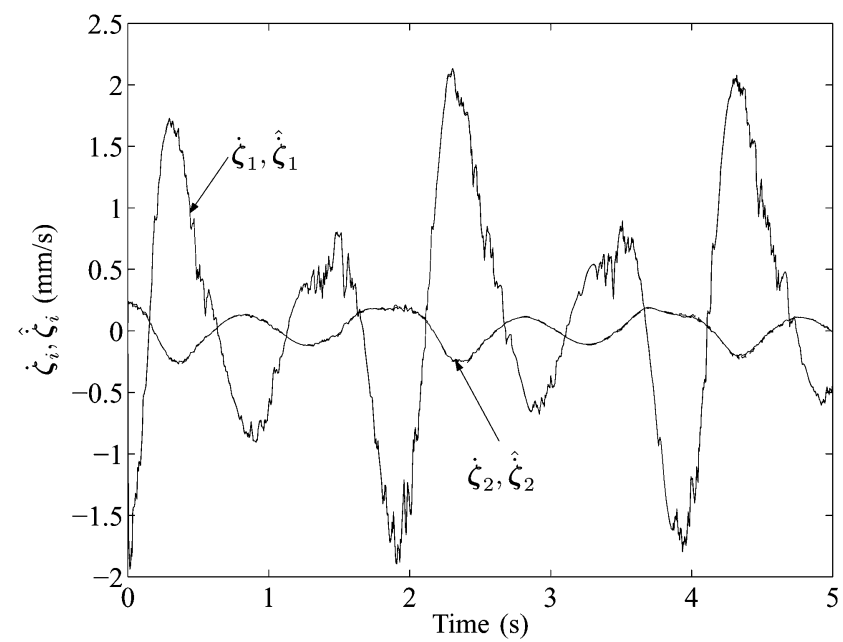

Fig. 9. Flexural generalized velocities; (solid) theoretical values and (dashed) estimated values.

estimator. Therefore, in a practical situation, if the system is modeled improperly, the accuracy of the results are expected to deteriorate. 


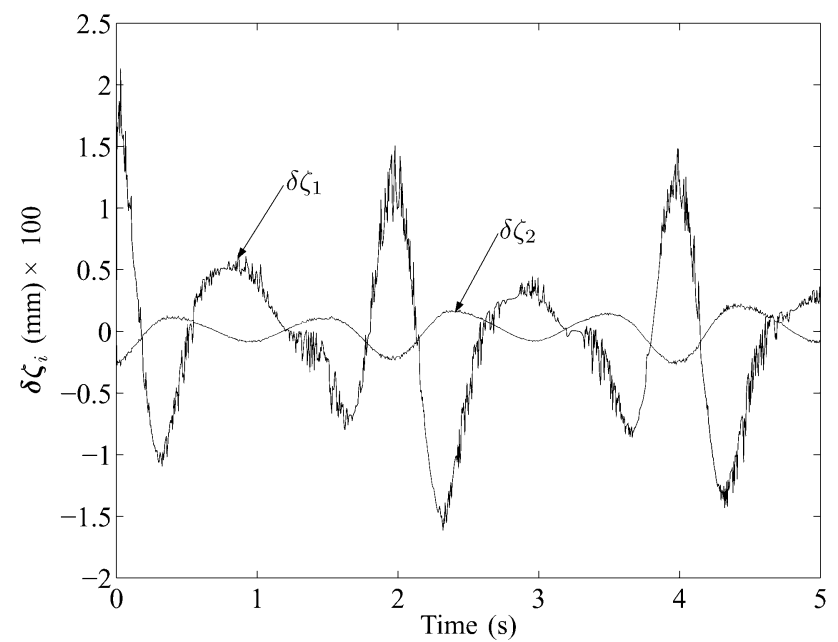

Fig. 10. Estimation error of the flexural coordinates.

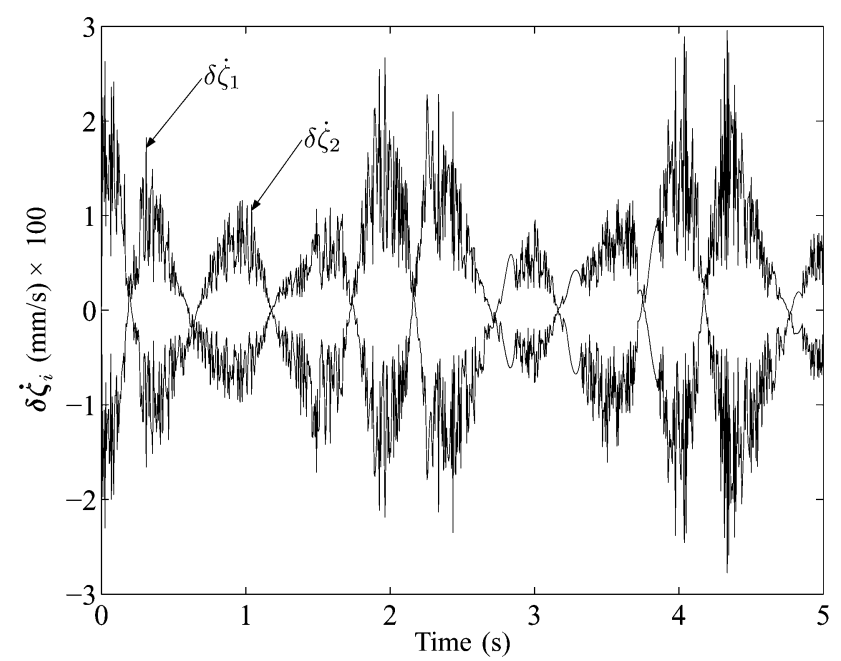

Fig. 11. Estimated error of the flexural generalized velocities.

\section{OBSERVABILITY OF THE FlEXURAL MOTION}

A complete observability analysis for the type of systems treated in this paper seems quite elusive, due to the nonlinear, highly complex nature of both dynamics equations and state-output relations. Nonetheless, to help us visualize how many flexural generalized coordinates one can estimate using one accelerometer array, consider a cantilever flexible link. If an accelerometer array is installed at the tip of such a link, the three components of the translational acceleration and those of the angular acceleration of the array, totaling six, can be used to estimate six flexural coordinates: Four flexural generalized coordinates describing bendings in two planes plus two flexural coordinates in each plane; one of the latter represents the torsional twist of the link, the other the elongation of the link axis. Consequently, if, in a particular case, more than two flexural coordinates are needed to effectively describe the bending of a link in one plane, then additional accelerometer arrays must be installed on the link along its length.

By the same token, if a flexible planar manipulator has more than two links, extra accelerometer arrays-each comprising more than two two-axis, or four single-axis, accelerometers-in addition to the one located at the tip of the end link, have to be installed on the distal end of the links to provide the estimator with enough information. The same should be done if the bending deformation of any of the links is to be discretized with multiple flexural coordinates. Otherwise, what the estimator will correctly estimate will be the translational and rotational motions of the end-point frame of the end-link. If additional accelerometer arrays are to be used, one can relate the twist of two subsequent link-end frames to simplify the state-output relations.

\section{CONCLUSION}

The estimation of the flexural states, the flexural generalized coordinates and generalized velocities, of the flexible links of a macro-micro manipulator was discussed in this paper. It was proposed that the data collected by an accelerometer array fixed to the micromanipulator base be used to infer the translational and angular accelerations of the base as well as its translational and angular velocities. The acceleration data were used to separate the dynamics of the macromanipulator from that of its micro counterpart. The velocity data, on the other hand, were taken as the outputs, and their relations to the flexural states were written and linearized in closed form. Then, having all the state-space equations in linearized form, we used them in an EKF to estimate the flexural states.

The estimation method explained here was based on the assumption that the dynamics of the system is exactly known. Therefore, dynamics modeling of the system, in general, and that of the macromanipulator, in particular, requires precise knowledge of the model parameters.

Simulation results reported in this paper demonstrate that the proposed estimation algorithm can indeed produce reliable estimates of the states. Although we have made use of an EKF to estimate the states, the state-space equations derived here can be used in other types of observers as well, especially since most of the observers devised for nonlinear systems are based upon linear state-output relations.

\section{REFERENCES}

[1] R. H. J. Cannon and E. Schmitz, "Initial experiments on the end-point control of a flexible one-link robot," Int. J. Robot. Res., vol. 3, no. 3, pp. 62-75, 1984.

[2] W.-W. Chiang, R. Kraft, and R. H. J. Cannon, "Design and experimental demonstration of rapid, precise end-point control of a wrist carried by a very flexible manipulator,' Int. J. Robot. Res., vol. 10, no. 1, pp. 30-40, Feb. 1991.

[3] P. T. Kotnik, S. Yurkovich, and Ü.Özgüner, "Acceleration feedback control for a flexible manipulator arm," in Proc. IEEE Int. Conf. Robotics and Automation, vol. 1, Philadelphia, PA, Apr. 1988, pp. 322-323.

[4] L. J. Alder and S. M. Rock, "Adaptive control of a flexible-link robotic manipulator with unknown payload dynamics," in Proc. American Control Conf., San Francisco, CA, Jun. 1993, pp. 2088-2092.

[5] — , "Experiments in control of a flexible-link robotic manipulator with unknown payload dynamics: An adaptive approach," Int. J. Robot. Res., vol. 13, no. 6, pp. 481-495, Dec. 1994.

[6] T. Fukuda and A. Arakawa, "Optimal control and sensitivity analysis of two links flexible arm with three degrees of freedom," in Proc. IEEE Conf. Decision Control, Tampa, FL, Dec. 1989, pp. 2101-2106.

[7] J. Carusone, K. S. Buchan, and G. M. T. D'Eluterio, "Experiments in end-effector tracking control for structurally flexible space manipulators," IEEE Trans. Robot. Automat., vol. 9, no. 5, pp. 553-560, Oct. 1993.

[8] D. W. Cannon, D. P. Magee, and W. J. Book, "Experimental study on micro/macro manipulator vibration control," in Proc. IEEE Int. Conf. Robotics and Automation, Minneapolis, MN, Apr. 1996, pp. 2549-2554. 
[9] M. Moallem, R. V. Patel, and K. Khorasani, "An observer-based inverse dynamics control strategy for flexible multi-link manipulators," in Proc. IEEE Conf. Decision Control, Kobe, Japan, Dec. 1996, pp. 4112-4117.

[10] A. Konno, M. Uchiyama, Y. Kito, and M. Murakami, "Configurationdependent vibration controllability of flexible-link manipulators," Int. J. Robot. Res., vol. 16, no. 4, pp. 567-576, Aug. 1997.

[11] Y. Sakawa, F. Matsuno, Y. Oshawa, M. Kiyohara, and T. Abe, "Modeling and vibration control of a flexible manipulator with three axes by using accelerometers," Adv. Robot., vol. 4, no. 2, pp. 119-137, 1990.

[12] F. Matsuno and Y. Sakawa, "A simple model of flexible manipulators with six axes and vibration control by using accelerometers," J. Robot. Syst., vol. 7, no. 4, pp. 575-597, 1990.

[13] A. S. Zaki and W. H. ElMaraghy, "A robust observer for flexible-link manipulators control," in Proc. American Control Conf., Seattle, WA, Jun. 1995, pp. 3334-3338.

[14] K. L. Hillsley and S. Yurkovich, "Vibration control of a two-link flexible robot arm," in Proc. IEEE Int. Conf. Robotics and Automation, Sacramento, CA, Apr. 1991, pp. 2121-2126.

[15] E. Sim and S. W. Lee, "Active vibration control of flexible structures with acceleration feedback," J. Guidance, Control, Dyn., vol. 16, no. 2 , pp. 413-415, Mar.-Apr. 1993.

[16] Z. Tiemin, L. Youwu, Y. Shaoze, Z. Qing, and Z. Haigen, "Comparative study on the acceleration feedback and the strain feedback of a flexible manipulator," in IEEE Int. Conf. Systems, Man, Cybernetics, vol. 2, Beijing, China, Oct. 1996, pp. 1031-1035.

[17] M. O. Tokhi and A. K. M. Azad, "Design and development of an experimental flexible manipulator system," Robotica, vol. 15, pp. 283-292, 1997.

[18] E. A. Misawa and J. K. Hedrick, "Nonlinear observers-a state-ofthe-art survey," J. Dyn. Syst., Meas., Control, vol. 111, pp. 344-352, 1989.

[19] C. C. de Witt and J.-J. E. Slotine, "Sliding observers for robot manipulators," Automatica, vol. 27, no. 5, pp. 859-864, 1991.

[20] R. Sanchis and H. Nijmeijer, "Sliding controller-sliding observer design for nonlinear systems," Eur. J. Control, vol. 4, no. 3, pp. 208-234, 1998.

[21] T. Yoshikawa, K. Harada, and A. Matsumoto, "Hybrid position/force control of flexible-macro/rigid-micro manipulator systems," IEEE Trans. Robot. Automat., vol. 12, no. 4, pp. 633-639, Aug. 1996.

[22] D. N. Nenchev, K. Yoshida, P. Vichitkulsawat, and M. Uchiyama, "Reaction null-space control of flexible structure mounted manipulator systems," IEEE Trans. Robot. Control, vol. 15, no. 6, pp. 1011-1023, Dec. 1999.

[23] K. Parsa, J. Angeles, and A. K. Misra, "Linearized kinematics for state estimation in robotics," in Advances in Robot Kinematics: Theory and Applications, J. Lenarčič and F. Thomas, Eds. Dordrecht, The Netherlands: Kluwer, 2002, pp. 39-48.

[24] A. A. Shabana, "Flexible multibody dynamics: Review of the past and recent developments," Multibody Syst. Dyn., vol. 1, pp. 189-222, 1997.

[25] R. Schwertassek, "Flexible bodies in multibody systems," in Computational Methods in Mechanical Systems: Mechanism Analysis, Synthesis, and Optimization. ser. NATO ASI Series, J. Angeles and E. Zakhariev, Eds. Berlin, Germany: Springer-Verlag, 1998, pp. 329-363.

[26] K. Parsa, J. Angeles, and A. K. Misra, "Attitude calibration of an accelerometer array," presented at the IEEE Int. Conf. Robotics and Automation, Washington, DC, May 2002.

[27] K. Parsa, "Dynamics, state estimation, and control of manipulators with rigid and flexible subsystems," Dept. Mech. Eng., Ph.D. dissertation, McGill Univ., Montreal, QC, Canada, 2003.

[28] L. E. George and W. J. Book, "Inertial vibration damping control of a flexible base manipulator," IEEE/ASME Trans. Mechatronics, vol. 8, no. 2, pp. 268-271, Jun. 2003.

[29] K. J. Åström and B. Wittenmark, Computer-Controlled Systems: Theory and Design, 3rd ed. Upper Saddle River, NJ: Prentice-Hall, 1997.

[30] C. K. Chui and G. Chen, Kalman Filtering With Real-Time Applications, 2nd ed, ser. Springer Series in Information Sciences. New York: Springer-Verlag, 1991.

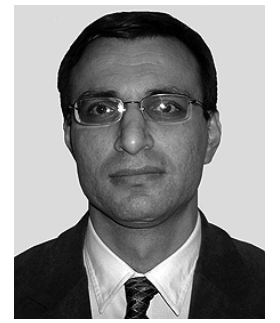

Kourosh Parsa received the B.S. and M.Sc. degrees in mechanical engineering from the Sharif University of Technology, Tehran, Iran, in 1989 and 1993, respectively, and the Ph.D. degree from McGill University, Montreal, QC, Canada, in 2003.

Before starting his doctoral studies, he worked for about four years on automated material handling systems at an automobile manufacturing company in Tehran, where he helped design the very first industrial automated guided vehicle in Iran from the ground up. He worked as a Postdoctoral Fellow at the University of California, Davis, from 2003 to 2004, on a suboptimal design of an all-accelerometer sensor array to be integrated in a GPS-assisted navigation system. His interests include dynamics modeling and control of structurally flexible multibody systems, space robotics, analytical dynamics, and multiaccelerometer sensor design.

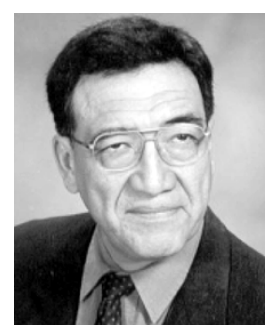

Jorge Angeles received the Engineer's diploma in electromechanical engineering and the M.Eng. degree in mechanical engineering from the National University of Mexico (UNAM), Mexico City, in 1969 and 1970, respectively, and the Ph.D. degree in applied mechanics from Stanford University, Stanford, CA, in 1973.

$\mathrm{He}$ is the author or coauthor of various books in the areas of kinematics and dynamics of mechanical systems, as well as numerous technical papers in refereed journals and conference proceedings. He focuses on the design and control of electromechanical systems. Besides his research activities, Angeles is a Consultant to various Canadian and international organizations on the matters of automation, mechanical design, and robotics; he also holds one U.S. patent, one Mexican patent, and has recently filed two patents on various PCT countries. He has held visiting positions in Germany and Singapore.

Dr. Angeles is a Fellow of the Royal Society of Canada, a James McGill Professor of Mechanical Engineering at McGill University, Montreal, QC, Canada; an NSERC Design Engineering Chair holder; an ASME Fellow; a Fellow of the Canadian Society for Mechanical Engineering; Past President of the International Federation for the Promotion of Mechanism and Machine Science (IFToMM); an IFToMM Honorary Member; and a member of various professional and learned societies. Professional registration as an engineer includes Quebec, Canada; Mexico; and Germany. He was a Nanyang Professor at Nanyang Technological Universit, Singapore, from 2000 to 2002, and he is an Honorary Professor at Tianjin University, Tianjin, China.

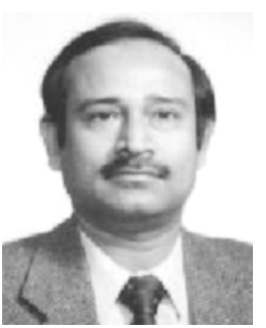

Arun K. Misra is the Chairman of the Department of Mechanical Engineering, McGill University, Montreal, QC, Canada. His research interests are in the areas of dynamics and control of multibody systems, particularly those encountered in space and in robotics.

Mr. Misra is a Fellow of the American Astronautical Society. 